\title{
Temperature Dependence of the Bragg Peak-Intensity Close to the $\alpha$-Incommensurate- $\beta$ Transition in Quartz
}

\author{
Hamit Yurtseven and Koray Kaymazlar \\ Department of Physics, Middle East Technical University, 06531 Ankara, Turkey \\ Correspondence should be addressed to Hamit Yurtseven; hamit@metu.edu.tr
}

Received 6 August 2014; Accepted 28 September 2014; Published 15 October 2014

Academic Editor: Víctor Lavín

Copyright (C) 2014 H. Yurtseven and K. Kaymazlar. This is an open access article distributed under the Creative Commons Attribution License, which permits unrestricted use, distribution, and reproduction in any medium, provided the original work is properly cited.

\begin{abstract}
Quartz as a mineral has a stable crystalline phase at room temperature and atmospheric pressure; at low temperatures it is in the $\alpha$ phase and when it is heated up, it transforms into the $\beta$ phase through the intermediate (incommensurate) phase within the temperature interval of nearly $1.3 \mathrm{~K}$ at around $847 \mathrm{~K}$. The order parameter $Q$ occurs due to a tilting of $\mathrm{SiO}_{4}$ tetrahedra around the threefold axis, which can be related to variation of the peak-intensity with the temperature in quartz. In this study, we analyze the temperature dependence of the Bragg peak-intensity measured through the $\alpha-\beta$ transition in quartz, as obtained from the literature according to a power-law formula. From our analysis, we deduce the values of the critical exponent $\beta$ for the order parameter (Bragg peak-intensity) for the $\alpha$-incommensurate (IC-) $\beta$ transition. Our $\beta$ values indicate that the $\beta$-IC phase transition is of a second order and that the IC- $\alpha$ phase transition is of a weak first order, as also reported in the literature.
\end{abstract}

\section{Introduction}

Quartz $\left(\mathrm{SiO}_{2}\right)$ is a mineral in crystalline form or amorphous [1] with various phases. At low temperatures in the $\alpha$ phase with symmetry $D_{3}$, it transforms into the $\beta$ phase with the symmetry $D_{6}[2]$ as the temperature increases. The transition temperature between the $\alpha$ and $\beta$ phases is about $847 \mathrm{~K}$. This crystalline structure was discovered by Le Chatelier in 1889 [3]. Since then, a great deal of work has been devoted to quartz. A review paper [4] and some studies on the $\alpha-\beta$ transition in quartz have been reported in the literature [59].

Measurements of the heat capacity [7-9] correlated with the thermal expansion have revealed the existence of an intermediate phase (incommensurate phase) in a small temperature interval of $\sim 1.3 \mathrm{~K}$ between the $\alpha$ and $\beta$ phases. We have analyzed the specific heat $[10,11]$ and we have examined the Pippard relations for the $\alpha-\beta$ transition in quartz [11] using the experimental data [8].

Spectroscopic studies on the $\alpha-\beta$ transition in quartz have also been reported in the literature. Some earlier Raman studies $[12,13]$ have investigated experimentally the soft mode behaviour of the $147 \mathrm{~cm}^{-1}$ and $207 \mathrm{~cm}^{-1}$ lattice modes close to the $\alpha-\beta$ transition in quartz. Neutron diffraction measurements $[6,7,14,15]$ have shown that the incommensurate phase is modulated.

Change in the symmetry from the $\alpha$ phase (low symmetry) to the $\beta$ phase (high symmetry) causes the order parameter $Q$ in the $\alpha$ phase to become zero in the $\beta$ phase. Ordering in the $\alpha$ phase is due to a tilting of $\mathrm{SiO}_{4}$ tetrahedra around the threefold axis. It has been pointed out [16] that the symmetry change gives rise to the excitation of many low-frequency high-amplitude modes of vibration which is associated with the phase transition in quartz. This can be the reason for the orientational disorder of the $\mathrm{SiO}_{4}$ tetrahedra and the order parameter for the displacive phase transition in quartz is then defined as the angle that each $\mathrm{SiO}_{4}$ tetrahedron rotates about the hexagonal or trigonal 100 directions [16]. In fact, it has been observed [17] that the infrared-active phonons near $795 \mathrm{~cm}^{-1}$ and $695 \mathrm{~cm}^{-1}$ and the Raman active mode near $355 \mathrm{~cm}^{-1}$ are associated with the symmetrybreaking order parameter of the $\alpha-\beta$ phase transition in quartz. This displacive transition in quartz is not a classical order-disorder transition since an orientational disorder still exists below $T_{c}$ in the $\alpha$ phase, which grows in the $\beta$ phase 
TABLE 1: Values of the critical exponent $\beta$ for the order parameter (Bragg intensity) and amplitude $A$ within the temperature intervals indicated for the $\alpha, \beta$ and incommensurate phases of quartz.

\begin{tabular}{|c|c|c|c|c|c|}
\hline Process & Phases & Temperature interval (K) & $2 \beta$ & $A\left[\mathrm{n} / \mathrm{s} \cdot \mathrm{K}^{2 \beta}\right]$ & Figure no. \\
\hline Heating & INC & $845.57<T<847$ & -0.35 & 13974 & Figure 1 \\
\hline Heating & INC & $843.95<T<845.3$ & -0.07 & 11003 & Figure 2 \\
\hline Heating & $\beta$ & $847.23<T<847.62$ & -0.53 & 8349 & Figure 3 \\
\hline Cooling & $\beta$ & $847.31<T<848.18$ & -0.27 & 4895 & Figure 4 \\
\hline Cooling & INC & $845.88<T<846.68$ & -0.29 & 10732 & Figure 5 \\
\hline Cooling & $\alpha$ & $843.61<T<843.95$ & 1.32 & 90219 & Figure 6 \\
\hline Cooling & $\alpha$ & $844.5<T<845.1$ & 0.62 & 26903 & Figure 7 \\
\hline
\end{tabular}

as the temperature increases [16]. It has been argued [16] that the dynamic disorder at the phase transition is not due to the fluctuations of the order parameter as in critical fluctuations, but it is due to the excitation of new low-energy vibrations, as stated above. It has also been argued [16] that the existence of the soft mode is not because of some degree of an order-disorder nature of the phase transition in quartz, but because of the excitation of low-energy vibrations which couple through anharmonic interactions with the soft mode. This soft mode which drives the phase transition in quartz propagates as a phonon that moves the $\mathrm{SiO}_{4}$ tetrahedra as rigid units [18]. A soft mode associated with a $\mathrm{SiO}_{4}$ rotation, which softens in the $\alpha$ phase, vanishes in the incommensurate phase and it hardens in the $\beta$ phase as the temperature increases. This has been the subject of a number of studies in quartz $[6,12,19-22]$. We have also studied the soft modes by calculating the Raman frequencies and the linewidths for the $\alpha-\beta$ transition in quartz $[23,24]$ and in $\mathrm{SiO}_{2}$ moganite [25].

In regard to the temperature dependence of the order parameter $Q$, in the $\alpha$ phase at low temperatures, variation of the order parameter related to the atomic displacements can be described by the variation of the peak-intensity with the temperature. The peak-intensity has been measured by neutron diffraction experiments [15] at various temperatures for the $\alpha-\beta$ transition in quartz.

In this study, we analyze the temperature dependence of the peak-intensity using the experimental data [15] by a power-law formula for the $\alpha-\beta$ transition in quartz. Below, we present our calculations and results in Section 2. In Section 3, we discuss our results and in Section 4 conclusions are given.

\section{Analysis and Results}

The temperature dependence of the intensity can be analyzed using a power-law formula close to the phase transitions. The divergence behaviour of the intensity at the transition point can be related to the order of parameter as

$$
Q^{2}(T) \sim I(T),
$$

where $Q(T)$ represents the temperature dependence of the order parameter below the transition temperature $T_{c}$. By describing this temperature dependence using a power-law formula

$$
Q \sim\left(T_{c}-T\right)^{\beta}
$$

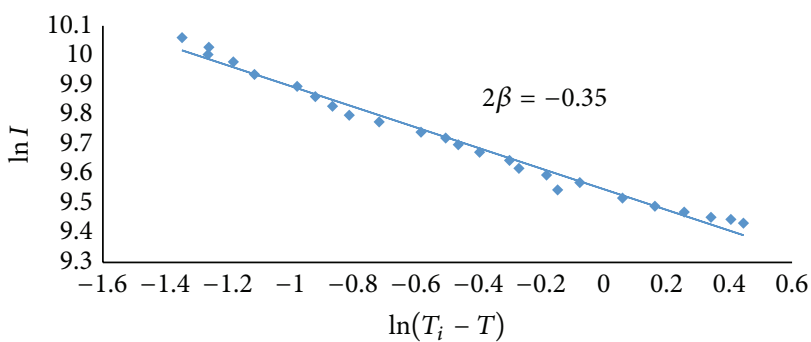

Figure 1: Peak-intensity as a function of temperature in a ln-ln scale in the incommensurate phase $\left(T_{i}=847.2 \mathrm{~K}\right)$ (heating) of quartz according to (4).

where $\beta$ is the critical exponent for the order parameter, the temperature dependence of the intensity can be expressed as

$$
I=A\left(T_{c}-T\right)^{2 \beta} .
$$

In (3), $A$ is the amplitude. By taking the logarithm of both sides, we obtain

$$
\ln I=\ln A+2 \beta \ln \left|T_{c}-T\right| .
$$

Thus, by knowing the temperature dependence of the intensity the values of $\beta$ and $A$ can be determined. Value of the critical exponent $\beta$ characterizes the phase transition of the system studied.

In this study, we analyzed the temperature dependence of the Bragg peak-intensity measured for the $\alpha-\beta$ transition in quartz [15] according to a power-law formula (3). This analysis was performed in the vicinity of the incommensurate phase $(\sim 1.3 \mathrm{~K})$ between $\alpha$ and $\beta$ phases for $T_{c}=T_{i}-4 \mathrm{~K}(\alpha$ phase $)$ and $T_{c}=T_{i}+4 \mathrm{~K}(\beta$ phase $)$, where $T_{i}=847.2 \mathrm{~K}$ as the incommensurate transition temperature of quartz.

From our analysis, we obtained the values of $\beta$ and $A$ for the $\alpha, \beta$ and incommensurate phases as given in Table 1. Figures 1 and 2 give in the heating process $\ln$-ln plots of Bragg intensity as a function of temperature in the incommensurate phase. For the $\beta$ phase, we also plot in the ln-ln scale the Bragg intensity at various temperatures for the heating and cooling processes, respectively, in Figures 3 and 4. For the cooling process, Figure 5 gives our plot of $\ln (I)$ versus $\ln \left(T_{c}-T\right)$ in the incommensurate phase of quartz. For this process, we plot $\ln I$ versus $\ln \left(T_{c}^{\prime}-T\right)$ for $\alpha$ phase in Figure 6. Finally, for the cooling process in the $\alpha$ phase $\ln I$ versus $\ln \left(T_{c}-T\right)$ is plotted in Figure 7. 


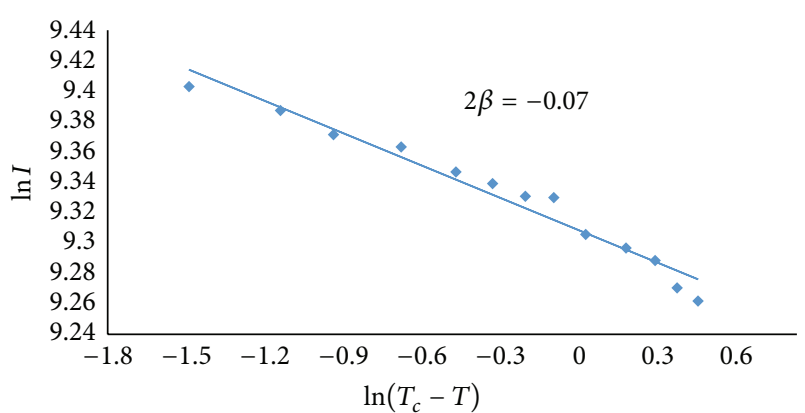

FIGURE 2: Peak-intensity as a function of temperature in a $\ln$-ln scale for the incommensurate- $\beta$ transition (heating) $\left(T_{c}=845.52 \mathrm{~K}\right)$ of quartz according to (4).

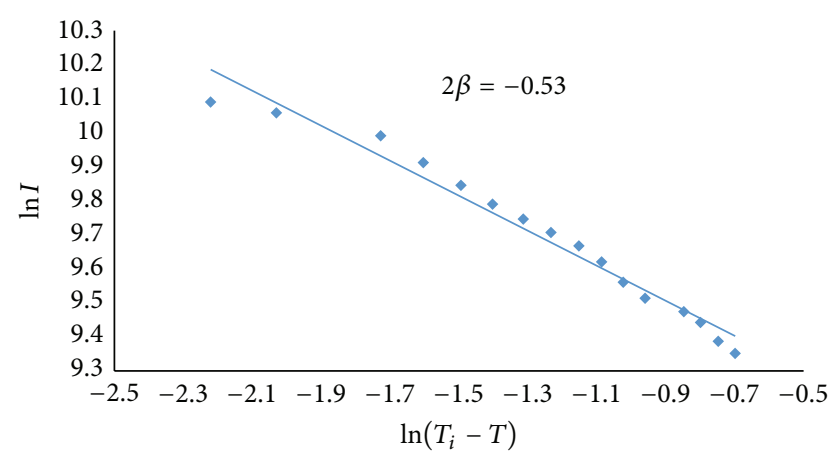

FIGURE 3: Peak-intensity as a function of temperature in a $\ln$-ln scale in the $\beta$ phase (heating) $\left(T_{i}=847.2 \mathrm{~K}\right)$ of quartz according to (4).

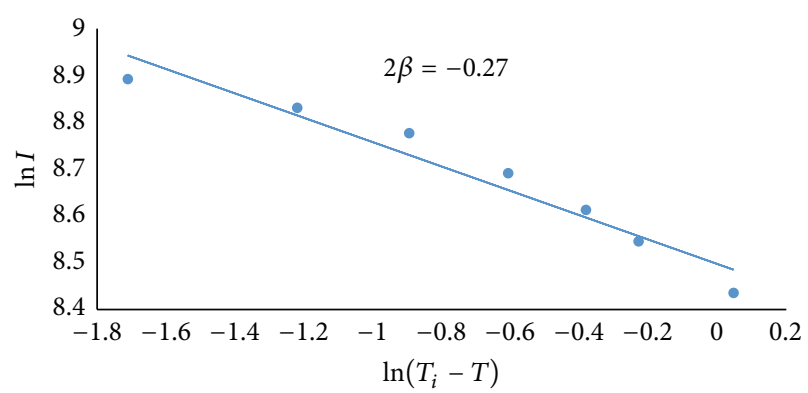

FIGURE 4: Peak-intensity as a function of temperature in a $\ln$-ln scale in the $\beta$ phase (cooling) ( $T_{i}=847.2 \mathrm{~K}$ ) of quartz according to (4).

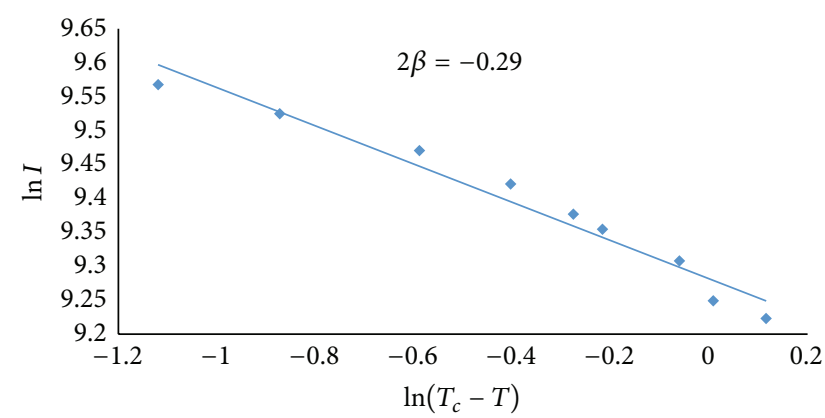

FIGURE 5: Peak-intensity as a function of temperature in a $\ln$-ln scale for the incommensurate- $\alpha$ transition (cooling) $\left(T_{c}=845.56 \mathrm{~K}\right)$ of quartz according to (4).

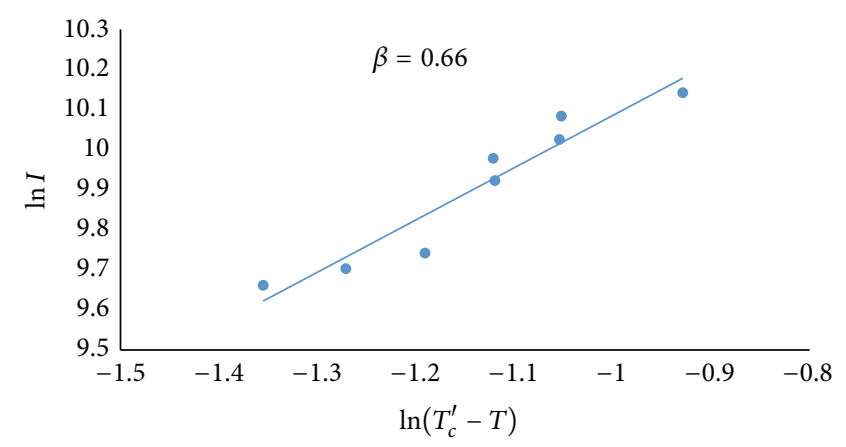

FIGURE 6: Peak-intensity as a function of temperature in a ln-ln scale in the $\alpha$ phase (cooling) $\left(T_{c}^{\prime}=843.59 \mathrm{~K}\right)$ of quartz according to (4).

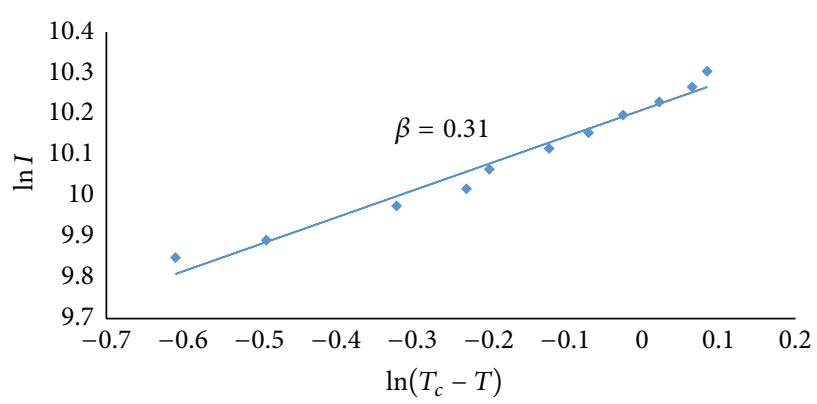

FIgURE 7: Peak-intensity as a function of temperature in a $\ln$-ln scale in the $\alpha$ phase (cooling) $\left(T_{c}=845.56 \mathrm{~K}\right)$ of quartz according to (4).

\section{Discussion}

The Bragg peak-intensity was analyzed here using the experimental data [15] according to a power-law formula (see (3)). Values of the critical exponent $\beta$ for the order parameter (Bragg peak-intensity) were determined for the $\alpha$ incommensurate- $\beta$ phase transition in quartz (Table 1). For the heating and cooling runs, values of $\beta$ and $A$ as given within the temperature intervals for the $\alpha$-incommensurate$\beta$ transitions vary from $2 \beta=-0.53$ to $\beta=0.62$. This large variety of the critical exponent $(\beta)$ characterizes first order and second order transitions occurring in quartz.

As noted previously [15], a second order transition occurs from the $\beta$ phase to the incommensurate phase at $T_{i}=$ $847.2 \mathrm{~K}$. The negative values from $2 \beta=-0.35$ to -0.07 (Table 1) below $T_{i}$ in the incommensurate phase should characterize a second order transition between the $\beta$ phase and incommensurate phase. This is due to the fact that the (022) Bragg peak-intensity diverges as $\left(T-T_{i}\right)^{-\beta}$ as the critical scattering intensity since this mode is overdamped and unstable which exhibits the soft mode behaviour. Similar soft mode behaviour has been observed [15] for the temperature dependence of the integrated scattering intensity for the $(1,0,3)$ reflection in the $\alpha-\beta$ quartz phase transition by inelastic neutron scattering as the Curie law divergence in ferroelectrics. This transition changes its character from a second order ( $\beta$ to INC phases) to the first order (INC to the $\alpha$ phases) at $T_{c}=845.56 \mathrm{~K}$. In the cooling process, the first order transition can be recognized by the values of the 
critical exponent which vary from $\beta=0.66$ to 0.31 (Table 1 ). Although the large value of the critical exponent $(\beta=0.66)$ indicates a first order transition, the value of 0.31 is close to the values of $0.326 \pm 0.004,0.38 \pm 0.03$, and 0.33 as predicted from the Ising $(d=3)$, Heisenberg $(d=3, D=3)$, and XY models [26], respectively, which describe the second order transition. This then indicates that, in the cooling process, transition from the incommensurate to the $\alpha$ phase at $T_{c}=845.56 \mathrm{~K}$ can be considered as a weak first order or close to the second order in quartz. This is not in disagreement with the earlier conclusion that the $\beta$ to the incommensurate transition at $T_{i}$ is found to be continuous, but the incommensurate to the $\alpha$ transition at $T_{c}$ is of first order [27]. The fact that the second order transition ( $\beta$ to incommensurate phase at $T_{i}$ ) changes to a weak first order transition (incommensurate to the $\alpha$ phase at $T_{c}$ ) may be due to a coupling of the order parameter (Bragg peak-intensity) with other degrees of freedom [28] such as acoustic phonons in the incommensurate phase where the soft phonon condensates inside the Brillouin zone, as also pointed out previously [27].

Different from the second order transition for the cooling process when the $\beta$ phase undergoes the incommensurate (INC) phase at $T_{i}=847.2 \mathrm{~K}$, it has been observed that the two phases coexist indicating a first order transition [14, 29]. By the intense light scattering [30], the existence of heterogeneous structure has been observed experimentally [31] from the $\alpha$ to the $\beta$ phase at $T_{i}$ and from the incommensurate to the $\alpha$ phase at $T_{c}$ in quartz. Incommensurate phase that occurs between $\alpha$ and $\beta$ phases in quartz similar to $\mathrm{NaNO}_{2}$ [32] can be investigated in some detail using various physical properties which can be obtained both experimentally and theoretically.

\section{Conclusions}

Temperature variation of the (022) Bragg peak-intensity was analyzed using the experimental data by a power-law formula for the $\alpha$-incommensurate- $\beta$ transition in quartz. Values of the critical exponent for the Bragg peak-intensity associated with the order parameter indicate that the $\beta$ to incommensurate transition $\left(T_{i}\right)$ is of the second order whereas incommensurate to the $\alpha$ transition $\left(T_{c}\right)$ is a weak first order (or close to the second order) type in quartz. Scattered Bragg peak-intensity in the incommensurate phase $(\sim 1.3 \mathrm{~K})$ can be attributed to the divergence of the fluctuation of the order parameter, which can be regarded as the critical opalescence of the soft mode in this phase of quartz.

\section{Conflict of Interests}

The authors declare that there is no conflict of interests regarding the publication of this paper.

\section{References}

[1] R. B. Sosman, The Phases of Silica, Rutgers University Press, New Brunswick, NJ, USA, 1965.
[2] R. W. G. Wyckoff, Crystal Structures, vol. 1, Interscience, New York, NY, USA, 1965.

[3] H. le Chatelier, "Sur la dilatation du quartz," Comptes-Rendus de l'Académie des Sciences, vol. 108, pp. 1046-1049, 1889.

[4] J. F. Scott, "Soft-mode spectroscopy: experimental studies of structural phase transitions," Reviews of Modern Physics, vol. 46, no. 1, pp. 83-128, 1974.

[5] H. Grimm and B. Dorner, "On the mechanism of the $\alpha-\beta$ phase transformation of quartz," Journal of Physics and Chemistry of Solids, vol. 36, no. 5, pp. 407-413, 1975.

[6] G. Dolino and J. P. Bachheimer, "Effect of the $\alpha-\beta$ transition on mechanical properties of quartz," Ferrolelectrics, vol. 43, pp. 7786, 1982.

[7] G. Dolino, J. P. Bachheimer, and C. M. E. Zeyen, "Observation of an intermediate phase near the $\alpha-\beta$ transition of quartz by heat capacity and neutron scattering measurements," Solid State Communications, vol. 45, no. 3, pp. 295-299, 1983.

[8] M. Matsuura, H. Yao, K. Gouhara, I. Hatta, and N. Kato, "Heat capacity in $\alpha-\beta$ phase transition of quartz," Journal of the Physical Society of Japan, vol. 54, no. 2, pp. 625-629, 1985.

[9] H. Yao and I. Hatta, "Phase transitions of quartz studied by a.c. calorimetry," Thermochimica Acta, vol. 266, pp. 301-308, 1995.

[10] H. Yurtseven and M. Desticioğlu, "Critical behaviour of the heat capacity near the $\alpha$ - $\beta$ phase transition in quartz," High Temperature Materials and Processes, vol. 32, no. 2, pp. 189-194, 2013.

[11] H. Yurtseven and S. Ates, "Pippard relations and the analysis of the specific heat for the $\alpha-\beta$ transition in quartz," International Journal of Chemistry, no. 2, pp. 48-59, 2013.

[12] S. M. Shapiro, D. C. O'Shea, and H. Z. Cummins, "Raman scattering study of the $\alpha-\beta$ phase transition in quartz," Physical Review Letters, vol. 19, no. 7, pp. 361-364, 1967.

[13] A. S. Pine and P. E. Tannenwald, "Temperature dependence of Raman linewidth and shift in $\alpha$-quartz," Physical Review, vol. 178, no. 3, pp. 1424-1430, 1969.

[14] G. Dolino, "Angular and polarization properties of light scattering at the $\alpha-\beta$ phase transition of quartz," Physica Status Solidi (A), vol. 60, no. 2, pp. 391-401, 1980.

[15] G. Dolino, J. P. Bachheimer, G. Berge, and C. M. E. Zeyen, "Incommensurate phase of quartz: I. Elastic neutron scattering," Journal de Physique Paris, vol. 45, no. 2, pp. 361-371, 1984.

[16] M. G. Tucker, M. T. Dove, and D. A. Keen, "Simultaneous analysis of changes in long-range and short-range structural order at the displacive phase transition in quartz," Journal of Physics Condensed Matter, vol. 12, no. 48, pp. L723-L730, 2000.

[17] E. K. H. Salje, A. Ridgwell, B. Guttler, B. Wruck, M. T. Dove, and G. Dolino, "On the displacive character of the phase transition in quartz: a hard-mode spectroscopy study," Journal of Physics: Condensed Matter, vol. 4, no. 2, pp. 571-577, 1992.

[18] M. T. Dove, M. Gamghir, and V. Heine, "Anatomy of a structural phase transition: theoretical analysis of the displacive phase transition in quartz and other silicates," Physics and Chemistry of Minerals, vol. 26, pp. 344-353, 1999.

[19] J. D. Axe and G. Shirane, "Study of the $\alpha-\beta$ quartz phase transformation by inelastic neutron scattering," Physical Review B, vol. 1, no. 1, pp. 342-348, 1970.

[20] U. T. Höchli and J. F. Scott, "Displacement parameter, softmode frequency, and fluctuations in quartz below its $\alpha-\beta$ phase transition," Physical Review Letters, vol. 26, pp. 1627-1629, 1971.

[21] G. Dolino and M. Valade, "Lattice dynamical behaviour of anhydrous silica," Reviews in Mineralogy and Geochemistry, vol. 29, pp. 403-431, 1994. 
[22] M. B. Smirnov and A. P. Mirgorodsky, "Lattice-dynamical study of the $\alpha-\beta$ phase transition of quartz: soft-mode behavior and elastic anomalies," Physical Review Letters, vol. 78, no. 12, pp. 2413-2416, 1997.

[23] M. Kurt and H. Yurtseven, "Temperature dependence of the Raman frequency shifts and the linewidths in the $\alpha$ phase of quartz," Balkan Physics Letters, vol. 19, Article ID 191042, pp. 362-368, 2011.

[24] M. C. Lider and H. Yurtseven, "Calculation of the raman linewidths of lattice modes close to the $\alpha$ - $\beta$ transition in quartz," High Temperature Materials and Processes, vol. 31, no. 6, pp. 741747, 2012.

[25] M. C. Lider and H. Yurtseven, "Temperature dependence of the Raman frequency of an internal mode for $\mathrm{SiO}_{2}$-moganite close to the $\alpha$ - $\beta$ transition," Journal of Thermodynamics, vol. 1 , no. 1 , Article ID 892696, 2012.

[26] J. C. Le Guillou and J. Zinn-Justin, "Critical exponents for the $n$-vector model in three dimensions from field theory," Physical Review Letters, vol. 39, no. 2, pp. 95-98, 1977.

[27] B. Berge, G. Dolino, M. Vallade, M. Boissier, and R. Vacher, "Incommensurate phase of quartz-II: brillouin scattering studies," Journal de Physique, vol. 45, pp. 715-724, 1984.

[28] A. P. Levanyuk and D. G. Sannikov, Soviet Physics, Solid State, vol. 18, p. 1122, 1976.

[29] S. M. Shapiro and H. Z. Cummins, "Critical opalescence in quartz," Physical Review Letters, vol. 21, no. 23, pp. 1578-1582, 1968.

[30] I. A. Yakolev, L. F. Mikheeva, and T. S. Velichkina, Soviet Physics Crystallography, vol. 1, p. 91, 1956.

[31] G. van Tendeloo, J. van Landuyt, and S. Amelinckx, "The $\alpha \rightarrow$ $\beta$ phase transition in quartz and $\mathrm{AlPO}_{4}$ as studied by electron microscopy and diffraction," Physica Status Solidi (A) Applied Research, vol. 33, no. 2, pp. 723-735, 1976.

[32] D. Durand, F. Denoyer, M. Lambert, L. Bernard, and R. Currat, "Etude par rayons X et par neutrons de la phase incommensurable du nitrite de sodium," Journal de Physique, vol. 43, pp. 149$154,1982$. 

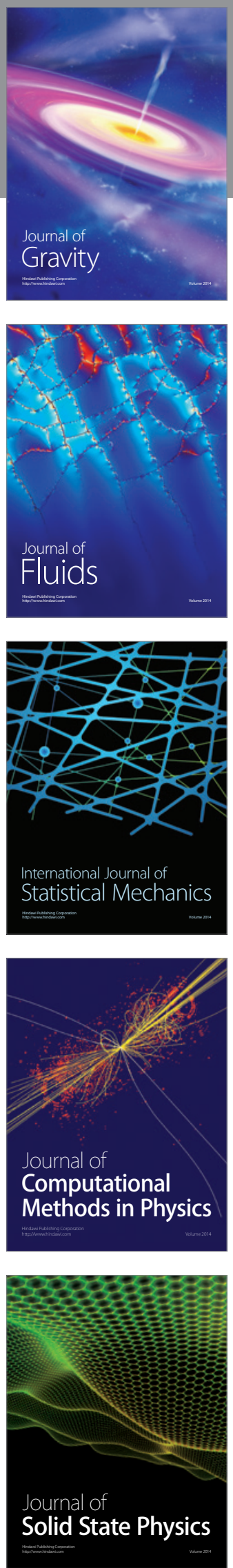

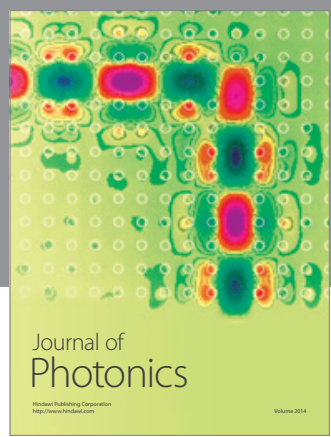

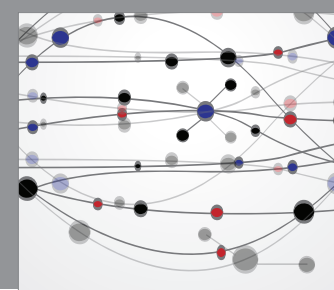

The Scientific World Journal

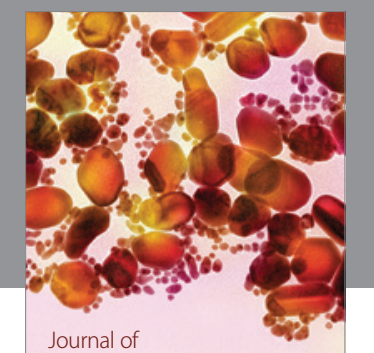

Soft Matter
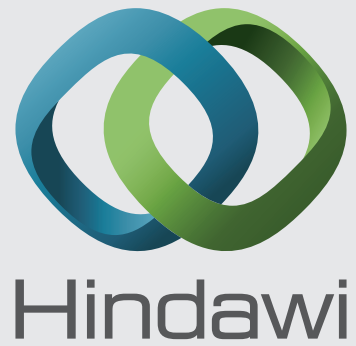

Submit your manuscripts at

http://www.hindawi.com
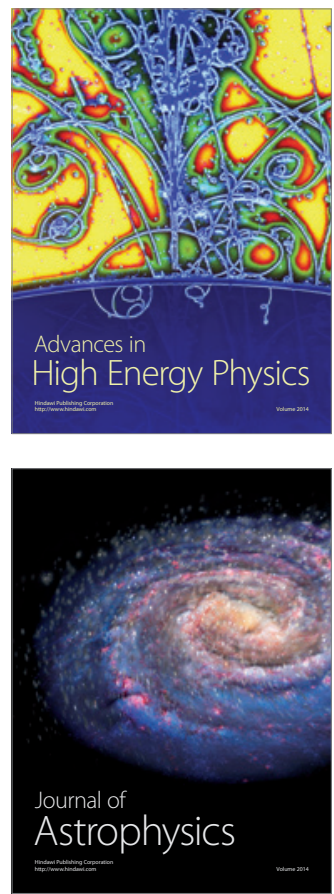
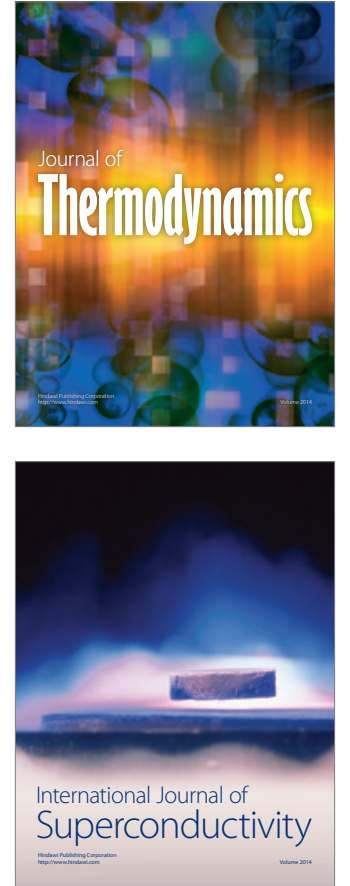
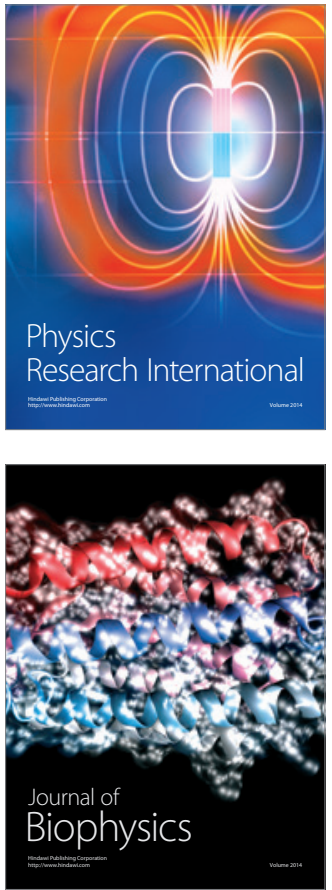
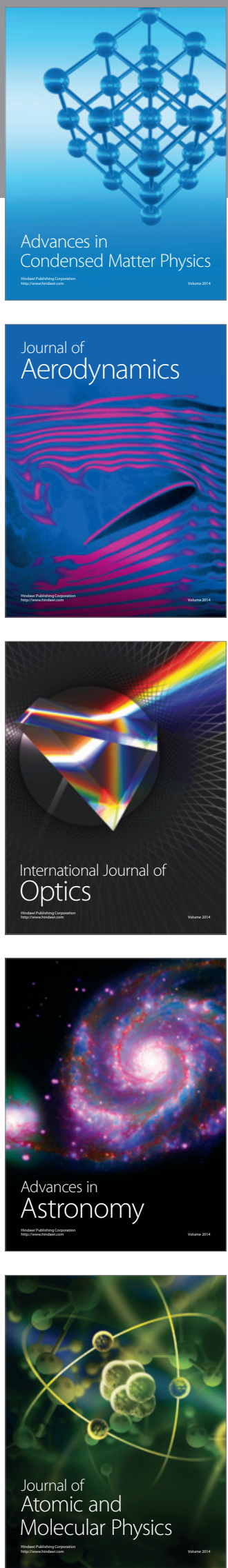